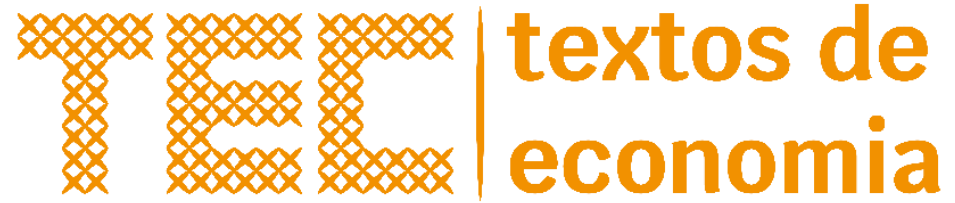

\title{
¿DONDE DEJO A MI HIJO? REDES PARA EL CUIDADO EM LA COMUNIDAD HISPANA DE NUEVA ORLEANS ${ }^{1}$
}

\section{Where should I leave my kid? Social Networks for the Care in the Hispanic} Community of Nueva Orleans

\author{
Diana Carolina Simbaña GONZÁLEZ \\ FLACSO Ecuador, Equador \\ nicarosi1@hotmail.com
}

\section{RESUMO}

Objetivo: Este artigo responde à pergunta. Quais são as estratégias que as famílias imigrantes ilegais monoparentais adotam em Nova Orleans para o cuidado de seus filhos mais novos (com menos de 5 anos)? Ele foca nas mulheres donas de casa como o único sustento da família; e aplica uma profunda entrevista à 7 mulheres que viviam em Nova Orleans no ano de 2017. Da perspectiva de que a economia vai além da análise do mercado, descobre-se que essas mulheres que assumem total responsabilidade por seus filhos usam modos diferentes de prover cuidados dos filhos e desenvolvem novas formas de combiná-los através do uso de seus contatos (acumulação de capital social). Essa tática possibilita sua conexão com a rede social de assistência comunitária hispânica. Essa aborgadem os ajuda a sobreviver nos EUA.

PALAVRAS-CHAVE: Nova Orleans. Cuidados. Redes Sociales. Capital Social.

\begin{abstract}
Objetive: This paper answers the question ¿Which are the strategies that monoparental undocumented immigrant families undertake in New Orleans for their youngest (less than 5 years old) children's care? It focuses on householder women as the only family sustain; and it applies in-depth interview to 7 women that live in New Orleans in the year of 2017 . From the perspective that economy goes farther than the analysis of the market, it finds that these women that take complete responsibility of their children use different provision modes for their children's care and develop new ways to combine them through the use of their contacts (accumulation of social capital). This tactic makes possible their connection with the hispanic community care social network. This approach helps them to survive in the USA.
\end{abstract}

KEYWORDS: New Orleans. Care. Social Networks. Social Capital.

Classificação JEL: B54

Recebido em: 12-02-2020. Aceito em: 08-06-2020.

\footnotetext{
${ }^{1}$ La siguiente investigación forma parte de la tesis de grado de mi autoría denominada "Tejiendo comunidad: estrategias de mujeres hondureñas para suplir servicios de cuidado en la ciudad de New Orleans" escrita como proyecto final de la maestría de Género y Desarrollo de Flacso Ecuador cursada en el período 20152017.
} 


\section{INTRODUÇÃO}

El cuidado para los niños pequeños menores de cinco años en los Estados Unidos es un asunto problemático, su organización está en crisis. Las vías de aprovisionamiento: Estado, mercado, familia, y comunidad; hacia donde se externaliza el cuidado no están dispuestos en igual medida para todas las personas, sino que su provisión está "condicionada siempre por su posición ${ }^{1}$ en la estructura económica y social en general" (Narotzky, 2007, p. 33). En este país, el Estado ofrece deficientes programas para su cobertura y los costos en el mercado son inaccesibles para la mayoría de las familias.

Quienes residimos en este país, entendemos la gravedad del asunto, y pese a que el problema se ha expuesto en la arena política en algunas ocasiones, todavía no se plantea ninguna política pública que garantice el cuidado de niños menores de 5 años que sea coherente con las necesidades de la población. El Estado, en la mayoría de los estados, únicamente otorga educación elemental y secundaria por ley durante seis horas diarias a niños mayores de 5 años. Si bien existen programas que proveen cuidado a niños pequeños (Head Start y Early Head Start por ejemplo), estos tienen serias restricciones y poco presupuesto para sostenerse; por lo tanto, benefician a un porcentaje muy mínimo de la población.

Por otro lado, las cifras por costo del cuidado en el mercado pueden llegar a ser tan exorbitantes que podrían estar en el rango de lo que se paga por la universidad e inclusive el precio llega a ser más alto que el promedio del costo de la renta de una vivienda (Child Care in America, 2014). A pesar de ello, la mayoría de familias con niños pequeños realizan esfuerzos enormes para comprarlo en el mercado, pues parecería que es la única vía dispuesta de aprovisionamiento.

La restricción en cuanto al cuidado se agudiza para las familias migrantes a las que se refiere esta investigación, pues el acceso depende del tipo de familia ${ }^{2}$ que lo requiera y su status legal (Menjivar, Abrego y Schalzbauer, 2016). Habitualmente, "los hogares migrantes quedan al margen de la deficiente red de servicios públicos de cuidados" (Pérez Orozco, 2009, p. 16), pues son víctimas del "bloqueo en el acceso a los beneficios públicos"

\footnotetext{
${ }^{1}$ De las familias.

2 El tipo de familias en el contexto migratoria también va a variar. Existe la posibilidad de que existan familias completas migrantes, cuyos integrantes tengan nacionalidades extranjeras y residan en los Estados Unidos. Así como también existen "familias mixtas" (Menjivar, Abrego y Schalzbauer, 2016, p. 140 refiriéndose a van Hook and Balistreri , 2006) cuyos integrantes portan diferentes status migratorios y nacionalidades. La posibilidad de accesos va a depender de las leyes de migración. En el capítulo siguiente se ampliará este tema.
} 
(Menjivar, Abrego y Schmalzbauer, 2016, p. 158) por no contar con documentación regularizada o pruebas de ciudadanía. Es decir, "se les vulnera su derecho al cuidado (Pérez Orozco, 2009, p. 15). En algunos casos, estas documentación no es requerida, sin embargo, aquellos que son migrantes sin documentación regularizada se auto limitan de acercarse a las instituciones públicas por miedo a poner en evidencia el estatus legal irregularizado de alguno de los miembros de la familia y ser deportados o poner en riesgo a sus familiares (Menjivar, Abrego y Schmalzbauer, 2016).

Para estas familias el cuidado se privatiza, pues: 1) se compra en el mercado, y 2) el lugar en donde se siguen gestionando la satisfacción del mismo es la familia a través de las estrategias de supervivencia (Pérez Orozco, 2009). De esta manera, "el cuidado sigue siendo una cuestión doméstica y no una responsabilidad traducida en la existencia de un derecho" (Pérez Orozco, 2009, p. 17). La imposibilidad de satisfacción de éste genera la llamada crisis de cuidados (Vega, 2009).

Las familias en las que se centra esta investigación son familias migrantes monoparentales encabezados por mujeres de nacionalidad hondureña que portan estatus legales irregularizados y tienen hijos menores de cinco años a su cargo, salieron de Honduras como estrategia de embate a la precariedad que vivieron en este país; y llegan a Estados Unidos luego de superar los inconvenientes propios de la movilidad e ingreso irregularizados. La hipótesis que se plantea indica que el aprovisionamiento del cuidado para ellas, es posible a través del aprovechamiento de vínculos (acumulación de capital social $^{3}$ ) (Bourdieu, 1985; Portes, 1999) que se encuentran desplegados en las redes que conectan a la comunidad hispana en los Estados Unidos. Estas mujeres externalizan el cuidado en espacios comunitarios. ¿Cómo lo hacen? Se verá a continuación.

El contexto geográfico específico en el que se realizó la investigación fue el área metropolitana de Nueva Orleans que es parte del estado de Luisiana. Esta área es considerada uno de los destinos más concurridos de los migrantes hondureños en los Estados Unidos pues históricamente se han construido fuertes conexiones entre ambos espacios que les permiten movilizarse con mayor facilidad (Euraque, 2004; Murillo, 2016).

\footnotetext{
${ }^{3}$ El capital social entendido como "agregado de los recursos reales o potenciales que se vinculan con la posición de una red duradera de relaciones más o menos institucionalizadas de conocimiento o reconocimiento mutuo" (Portes, 1999, p.3 citando a Bourdieu, 1985, p. 248), o la capacidad "de movilizar recursos de diversos tipos en función de la pertenencia a una red" (Pozas, 2007, p. 631-632 citando a Portes, 1995).
} 


\section{MÉTODOLOGÍA}

El enfoque metodológico utilizado en esta investigación fue de orden cualitativo. Las herramientas que se usaron para la reconstrucción de las estrategias familiares para el aprovisionamiento del cuidado en Nueva Orleans metro-área fueron: 1) la observación participante en eventos de interés de la comunidad hondureña en Nueva Orleans como la fiesta de la virgen de Suyapa "La Morenita", o el concierto del Grupo Isieni en el carnaval de la Ceiba en Nueva Orleans, en donde se estableció contacto con actores de la comunidad. Además, se visitaron espacios de concurrencia latina como restaurantes latinos y hondureños, el Apostolado Hispano, el Consulado de Honduras en Nueva Orleans, parques y mercados que ofertan productos hispanos, en donde también se contactaron personas de origen hondureño que guiaron la comprensión de cómo se satisface el cuidado fuera del Estado y el mercado en Nueva Orleans; y, 2) se aplicaron entrevistas a profundidad a siete mujeres que se constituyen en cabezas de hogar de las familias con las características ya mencionadas; además, se aplicaron entrevistas semiestructuradas a cuatro miembros de organizaciones influyentes de la red migratoria hispana en Nueva Orleans.

La observación participante se realizó entre noviembre del 2016 y abril del 2017 y las entrevistas se realizaron entre febrero y abril del 2017. La información obtenida de las entrevistas fue organizada en torno a los vínculos que se detectaron que fueron facilitadores del acceso al cuidado. Para establecer estos vínculos se recurrió a la propuesta teórico metodológico que ofrece Claudia Pedone (2010) que indica que el "análisis de los vínculos 'fuertes' y 'débiles', y las relaciones 'horizontales' y 'verticales' que articulan la dinámica de las cadenas y redes migratorias" (Pedone, 2010, p. 111) dilucida el papel que los actores desempeñan dentro de la red, así como los vínculos que se forman en torno a los bienes dispuestos en ellas.

Todas las entrevistas fueron grabadas en formato de audio, todas las mujeres indicaron sus nombres sin apellido y dieron autorización para difundir sus experiencias. Por otra parte, las entrevistas a los miembros de organizaciones influyentes fueron tomadas en cuenta en los casos oportunos en el análisis de resultados de esta investigación identificados con un pseudónimo.

A continuación presento la información general de las entrevistadas: 
Tabla 1 - Caracterización socioeconómica de las mujeres que participaron en la investigación

\begin{tabular}{|c|c|c|c|c|c|c|c|c|c|}
\hline Nombre & Edad & $\begin{array}{l}\text { Fecha llegó a } \\
\text { New Orleans }\end{array}$ & $\begin{array}{c}\text { \# de hijos } \\
\text { menores de } \\
18 \text { años }\end{array}$ & $\begin{array}{c}\text { \# de hijos } \\
\text { menores de } \\
\text { cinco años que } \\
\text { reciben cuidado } \\
\text { fuera de casa }\end{array}$ & $\begin{array}{c}\text { Ingreso Promedio/ } \\
\text { Depende de } \\
\text { cuánto trabajo } \\
\text { haya }\end{array}$ & $\begin{array}{c}\text { Nivel de } \\
\text { instrucción }\end{array}$ & $\begin{array}{c}\text { Fluencia en el } \\
\text { idioma inglés }\end{array}$ & Ocupación & $\begin{array}{c}\text { Subsidio, bono o } \\
\text { servicio del } \\
\text { Estado que recibe }\end{array}$ \\
\hline Suli & 28 & 2014 & 1 & 1 & $\begin{array}{c}\text { entre } \$ 8 \text { y } \$ 12 \\
\text { /hora }\end{array}$ & $\begin{array}{l}\text { secundaria } \\
\text { inconclusa }\end{array}$ & bajo & $\begin{array}{c}\text { limpieza de } \\
\text { casas, } \\
\text { portera }\end{array}$ & ninguno \\
\hline Gertrudis & 36 & 2009 & 3 & 1 & $\$ 12$ hora & $\begin{array}{l}\text { secundaria } \\
\text { inconclusa }\end{array}$ & bajo & $\begin{array}{c}\text { limpieza en } \\
\text { instituciones }\end{array}$ & ninguno \\
\hline Yajaira & 31 & 2007 & 4 & 1 & $\begin{array}{c}\text { entre } \$ 8 \text { y } \$ 12 \\
/ \text { hora }\end{array}$ & $\begin{array}{l}\text { secundaria } \\
\text { completa }\end{array}$ & alto & \begin{tabular}{|c|} 
dueña de \\
compañía de \\
limpieza
\end{tabular} & $\begin{array}{l}\text { SNAP conocida } \\
\text { como Food Stamps }\end{array}$ \\
\hline Beatriz & 55 & 2006 & $\begin{array}{c}1 \text { (nieta a } \\
\text { cargo) }\end{array}$ & 1 & $\$ 300$ semanales & primaria & bajo & \begin{tabular}{|c|} 
ayudante de \\
cocina
\end{tabular} & ninguno \\
\hline Vanesa & 28 & 2009 & 2 & 1 & $\$ 12$ hora & primaria & bajo & $\begin{array}{c}\text { limpieza de } \\
\text { habitaciones } \\
\text { de hotel }\end{array}$ & $\begin{array}{l}\text { SNAP conocida } \\
\text { como Food Stamps }\end{array}$ \\
\hline Jasel & 26 & 2011 & 3 & 1 & $\$ 8$ & secundaria & medio & $\begin{array}{c}\text { lava platos, } \\
\text { mesera }\end{array}$ & $\begin{array}{l}\text { SNAP conocida } \\
\text { como Food Stamps }\end{array}$ \\
\hline Yiudmila & 46 & 2005 & 2 & 1 & $\begin{array}{c}\$ 20 \text { diarios por } \\
\text { niño }\end{array}$ & primaria & bajo & $\begin{array}{c}\text { cuidado de } \\
\text { niños, } \\
\text { limpieza de } \\
\text { casas }\end{array}$ & $\begin{array}{l}\text { SSI Seguro de } \\
\text { Ingreso } \\
\text { Suplementario }\end{array}$ \\
\hline
\end{tabular}

Fuente: Diarios de Campo (Febrero-Abril, 2017).

\section{RESULTADOS}

A pesar de la crisis, el cuidado constantemente "se reorganiza en y entre familias, géneros y clases, pero también entre iniciativas privadas, públicas y del sector cívico asociativo" (Vega 2009, 49). Para esta reorganización, los individuos y grupos crean cada vez nuevas "prácticas efectivas de aprovisionamiento" (Narotzky 2007, 35) que dependen de "la cambiante capacidad individual o doméstica de articular redes sociales" (Narotzky 2007, 27). A continuación los ejemplos de cómo lo hacen.

\section{Las Cuidadoras de Drupo}

Los ingresos de las mujeres entrevistadas son limitados, no dan abasto para la externalización del cuidado en el mercado en guarderías privadas o centros de cuidado de iglesias que abundan en la ciudad. Sin embargo, existen opciones informales que como indica Narotzky (2007, p. 27) se encuentran dentro de las "posibilidades no-mercantiles (y) pueden estar parcialmente insertadas en el intercambio de mercado". Estas no se 
publicitan, sino que más bien son conocidas por las personas que están inmersas en la red, y su información se pasa de "de boca en boca". Se trata de la oferta de servicios de otras mujeres en condiciones similares de vulnerabilidad, que como estrategia de supervivencia abren las puertas de su casa para recibir niños y cuidarlos a cambio de una remuneración muy inferior a las que requieren los centros formales. Este es el caso de Yiudmila:

Pues mire aquí al salario hablando de mi les cobro 20 dólares si yo les cuido
todo el día y ellos me traen la comida. Claro yo siempre les doy de lo mío,
pero yo le pongo así a la mama para que tenga un poquito de
responsabilidad porque si yo le digo tráigale a la bebe debe 20 dólares y ya
yo tengo que reponer la manzana tengo que poner esto y otro porque
imagínese son tres y con los míos son 5 , y si quieren que yo les de la comida
les cobro 5 dólares más. Hasta ahorita ninguno me está dando 5 dólares
más por lo que yo les voy a dar de comer pero ya ahí es su desayuno
almuerzo y cena en realidad por 5 dólares con toda la merienda que es los
snacks. Si no tiene los 5 dólares entonces que tenga la responsabilidad de
ponerles todo lo que el niño va a comer, su desayuno, su almuerzo y sus
snacks y si me traen toda la semana de lunes a sábado. Si el papa solo
trabajo de lunes a viernes porque estuvo lloviendo ese día no se lo cobro, a
mí solo me pagan los días que vienen \{...\} Ahorita tengo al más chiquito que
es Víctor de 2 añitos, Sibelis tiene 6 y Willy tiene 5 (Yiudmila, comunicación
personal, 1 de abril del 2017 ).

En la comunidad hispana se sabe que hay mujeres que ofrecen este servicio. Sin embargo, no es posible llegar a ellas de manera sencilla. Es necesario tener algún contacto que facilite el vínculo con esta persona. Su oferta no es explícita porque su servicio no cuenta con la licencia que requiere el estado para cuidar niños en casas, en In-home daycares (cuidado de niños en casas que requiere un permiso otorgado por el Departamento de Educación de los Estados Unidos), por lo que su funcionamiento es ilegal.

Suli, por ejemplo, llega a Yiudmila a través de la iglesia "Emmanuel" a la que ella asiste. Una de las "hermanas" (en la fe) de su iglesia vive en el barrio de Yiudmila, en donde es conocida desde el huracán Katrina por brindar servicios de cuidado. Cuando Suli se separa quedando sola con su niño, Felicia (vecina de Yiudmila) se solidariza brindándole la información de Yiudmila, mostrando así los "lazos de cooperación y solidaridad en los lugares de origen y llegada" (Pedone, 2004, p. 231) que se dan en algunas relaciones horizontales en las iglesias.

Suli delega el cuidado de su hijo Victor a Yiudmila desde los 3 meses de edad. Ella sostiene que depende de Yiudmila para poder trabajar y destaca la flexibilidad de su servicio que es beneficiosa para ella. Suli indica que ha dejado a Víctor hasta por doce horas diarias en la casa de Yiudmila pagando $\$ 20$ por día. 
Asimismo Jasel, que usa este tipo de servicio para el cuidado de su hijo, manifiesta que su jefa, dueña de un emprendimiento de comida en el mercado "La Pulga", le indica que la madre de la señora dueña del bazar es cuidadora y que debe acercarse a requerir sus servicios por su recomendación. Jasel hace contacto, la señora acepta y actualmente esta persona recibe a su hija por esta recomendación con un costo inferior al que cobra Yiudmila. Jasel declara que puede encargarla únicamente en las mañanas porque "la señora que la cuida trabaja en las tardes en otro sitio" (Jasel, comunicación personal, 13 de abril del 2017) y que a cambio le paga $\$ 50$ al mes más la cuenta de su plan de celular que es de $\$ 35$.

La pulga se convierte en un espacio de reunión en donde se tramitan bienes y servicios no sólo entre clientes y emprendedores sino también entre los mismos emprendedores que forman parte de la comunidad hispana. La gente que trabaja ahí se conoce, compra los artículos que necesitan a los otros emprendedores y también servicios como el cuidado. Como pasa con Yiudmila, en la pulga no hay ningún rótulo que ofrezca cuidado, pero el contacto diario de las personas en este lugar permite pasar de "boca en boca" la información de las cuidadoras.

Asimismo, Beatriz delega el cuidado de su nieta Joseline a Teresa, e indica que quien le introdujo a esta cuidadora fue F. Sánchez, una persona influyente en la comunidad hispana pues es miembro activo de la "Asociación de Hondureños Unificada de Luisiana" y forma parte de una fundación religiosa muy importante para los hondureños en Nueva Orleans denominada "Damas de Suyapa". Esta persona promueve eventos sociales y culturales en la ciudad, y además en su calidad de dirigente y activista genera el encuentro de los hondureños que viven en la ciudad. Beatriz señala que su presencia persistente en estos eventos y su fe hacia "La Morenita" le ayudaron a acercarse a este personaje que le facilitó el contacto para el cuidado. La congregación de Beatriz en eventos organizados por la comunidad hispana/ hondureña le permite dar a conocer su necesidad de cuidado y satisfacerla.

"La Morenita" como sus seguidores conocen a la virgen "Santa María de Suyapa" es la patrona de Honduras y los hondureños residentes en Nueva Orleans conmemoran su aparición la primera semana de febrero de cada año en la ciudad con una gran fiesta que es promovida por diferentes actores de la comunidad hispana como la Fundación Damas de Suyapa ${ }^{4}$, el Apostolado Hispano, el Consulado General de Honduras entre otras

\footnotetext{
${ }^{4}$ Las Damas de Suyapa realizan eventos para recolectar fondos y financiarla. La importancia de la fiesta es notoria pues todos los años cuenta con la presencia del Cónsul de Honduras en la ciudad, sacerdotes y
} 
organizaciones seculares y religiosas. En el 2017, se celebró con una misa precedida por el padre Juan Ángel López, obispo portavoz de la arquidiócesis de Tegucigalpa. Luego se dio lugar un banquete de comida típica hondureña en la cafetería de la iglesia que congregó a la comunidad (Diario de campo, 3 de febrero del 2017).

Generalmente, los que llegaron primero se "convierten en actores con cierta autoridad" (Pedone, 2004, p. 238). F. Sánchez es un ejemplo de este tipo de actores. Ella llega a Nueva Orleans a finales de los años 70. A través de las organizaciones que ha creado conoce a personajes de diferentes generaciones de hondureños y hace el papel de contacto puente entre estas personas. La relación de Teresa y Beatriz es un ejemplo de ello.

En las experiencias de Suli, Jasel y Beatriz, la cuidadora que satisface el cuidado de sus familias es una mujer que también habla español, también es indocumentada y su trabajo está inmerso en las actividades remuneradas de reproducción en condiciones precarizadas. La diferencia entre las cuidadores en el caso de Yiudmila y Suli, y la mamá de la señora del bazar del mercado "La Pulga" y Jasel, es la edad. Las cuidadoras son mayores de 45 años y estas entrevistadas son menores de 30 años. Esto podría indicar que el cuidado en casas podría ser una estrategia de las mujeres que no tienen ofertas de trabajo en otros mercados por ser estar en edades avanzadas.

\section{¿Cómo es posible ingresar en los programas públicos de cuidado? Los vínculos con instituciones que ostentan autoridade}

Pese a que existen programas de cuidado ofertados por el gobierno como el Head Start y Early Head Start, hay pocos hispanos que acceden a sus beneficios. A pesar de que uno de los requisitos para el acceso a los programas es probar que la familia recibe ingresos por debajo del límite de pobreza dispuesto en distintas cantidades para cada estado, y la población hispana tiene un alto porcentaje de precarización; esta población es una de la que menos accede a los programas públicos de cuidado. En Head Start de Luisiana, por ejemplo, sólo el $4 \%$ de hispanos de menos de cinco años se benefician del programa (Barnett y Friedman-Krauss, 2016). A continuación dos gráficas que ilustran esta información:

dirigentes de la arquidiócesis y otras personalidades como enviados de los canales locales de televisión dirigidos a la comunidad hispana como Telemundo (Diario de campo, febrero 2017). 
Gráfico 1 - Participación de los programas públicos de educación preescolar

Preschool participation by ethnicity

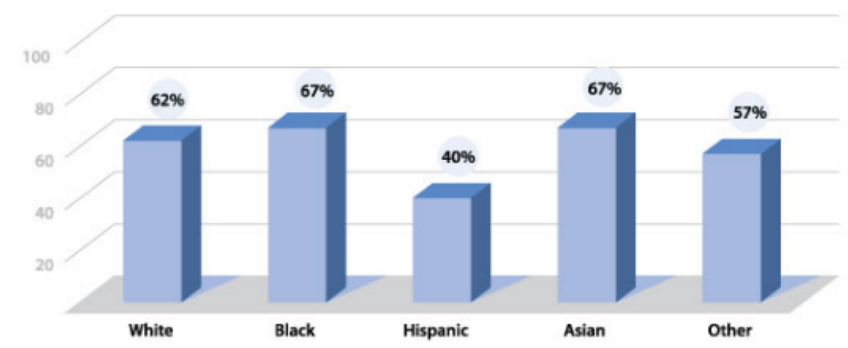

Fuente: Herchinger Report (marzo 17, 2010).

Gráfico 2 - Características del niño y la familia beneficiarias de Head Start y Early Head Start en Louisiana

LOUISIANA Head Start \& Early Head Start

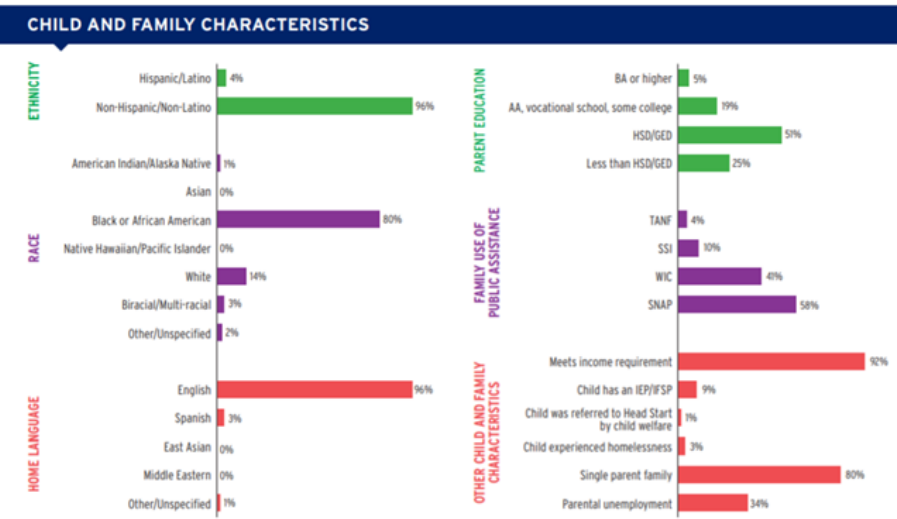

Fuente: The National Institute for Early Education Reseach (2016).

Las formas en las que el este $4 \%$ de hispanos accede al cuidado en Luisiana no son exploradas en esta investigación, sin embargo, en Nueva Orleans metro-área se observa un caso que explica la obtención de cuidado proporcionado por el Estado a través de la creación de un vínculo fuerte (cercano) con otro actor con autoridad en la red migratoria para el cuidado. Esto podría explicar la importancia para los migrantes de formar parte de una red, así como la creación y el aprovechamiento de vínculos a la hora de aprovisionarse de bienes necesarios. Se trata del Apostolado Hispano y uno de sus integrantes J. Maldonado. 
“El Apostolado Hispano abre sus puertas el 10 de marzo de 1972” (J Maldonado, comunicación personal, 15 de marzo del 2017) para ofrecer consejería y asistencia a los hispanos en asuntos sociales, religiosos y legales ${ }^{5}$ y se convierte en uno de los espacios de acogida para los hispanos/latinos recién llegados a Nueva Orleans, pues aquí se congregan organizan y forman comunidad. Con el paso del tiempo, el Apostolado fue cobrando fuerza, y se convirtió en un actor con autoridad en la red migratoria, y hoy por hoy ostenta una "posición privilegiada" (Pedone, 2004, p. 238). Por haber acumulado capital social cuenta con vínculos con, por ejemplo, entidades del Estado, que conducen a bienes y servicios como el cuidado. Sus integrantes como J. Maldonado tienen la función de ser contactos puentes, pues generan vínculos con otras instituciones, organizaciones y personas en todos los ámbitos de la comunidad.

Gertrudis crea un vínculo fuerte ${ }^{6}$ a través de relaciones horizontales (lazos de cooperación y solidaridad) (Pedone, 2004) con esta persona, así:

A mí me trajo es J Maldonado, y yo lo conocí a él pues viniendo a las misas hispanas. Yo lo seguí y el me encomendó pues a ser parte de los carismáticos porque me vio que yo le seguí con fe. Ahí fue que ya vamos años adorando y alabando. Por medio de él es que pude poner a mi hija en el daycare, sino fuera el caso, es difícil, bien difícil. Aquí todo es muy caro y yo aunque gracias a Dios tengo trabajo no me da para pagar tanto (Gertrudis, comunicación personal, 17 de marzo del 2017).

Gertruidis (2017) menciona que J. Maldonado le facilita el contacto y la recomendación para inscribir a su hija en la guardería "Incarnate Word/ Palabra encarnada" a tiempo completo. Gertrudis no porta documentos regularizados pero su hija es ciudadana

\footnotetext{
${ }^{5}$ El Apostolado Hispano otorga servicios como son "misas, confesiones, unciones, retiros espirituales, estudio bíblico, renovación carismática, preparación de celebraciones especiales (bautizos, primeras comuniones, matrimonios), servicios de consejería, entre otros asuntos de carácter espiritual", pero también ofrecen servicios "en la parte social hemos brindado servicios de inmigración, de refugiados, de asistencia inmediata, de ayuda a búsqueda de empleos. Hemos tenido ferias de la salud, hemos tenido ferias de trabajos, educación a través de vínculos con el gobierno local y federal para que la gente aprenda derechos y obligaciones, clases de ciudadanía, hemos tenido clases de defensa personal con la policía de Kenner para que las mujeres aprendan a defenderse ante casos de agresión física, tenemos también ayuda para los niños de primero a quinto grado y organizamos algo que se llama "regreso a la escuela" que se hace más o menos la última semana de julio donde mil niños pueden venir a llevar su back pack con todos los útiles escolares que piden en las escuelas de primero a quinto de primaria. Les hemos ayudado con uniformes, hemos ayudado incluso a algunos con becas para ingresar a colegios católicos y hay un programa estatal que se llama "ARETE" y hemos indicado a las personas cómo lo pueden llevar adelante, cómo pasar de educación pública a privada. Por mucho tiempo ayudamos a la gente con sus declaraciones de impuestos, y cómo adquirir un ITIN number, entre tantos otros servicios que hemos dado, sí" (J. Maldonado, comunicación personal, 15 de marzo del 2017)

${ }^{6}$ Esta característica de fuerza se da por "el reconocimiento de relaciones recíprocas y no por el hecho de que los individuos estén físicamente próximos" (Ramella, 1995, p. 20 citado en Sanchez y Zubrzycki, 2015).
} 
estadounidense, por lo que accede al programa Head Start. Gertrudis indica no haber tenido información del programa antes de su vinculación con J. Maldonado, sino que se entera de su existencia a partir de la información que éste le provee. En la actualidad, Gertrudis entiende que la guardería pública a la que envía a su hija tiene limitación de cupos y agradece recibir el apoyo en la aplicación y el ágil ingreso, pero no se percata de cuán privilegiada es en la comunidad hispana al aprovisionarse de este servicio.

Yo mencioné en una experiencia en la adoración, se lo pedí a mis hermanos, que necesitaba el apoyo para poder meter a mi hija a la escuelita, a mí no me alcanza el tiempo. Yo la dejaba con mis hijos mayores cuando tenía que trabajar pero, en las mañanas la tenía que cuidar yo misma y no me alcanzaba el dinero porque el rail y la renta asimismo son muy caros. Yo tenía que trabajar más tiempo, eso de las noches ya me estaba entrando el miedo dejar a mis hijos solos con la niña (Gertrudis, comunicación personal, 17 de marzo del 2017).

Yo agradezco a J. Maldonado, que es tan fiel y ayudador. Mejor ahora que vivo de día, no es cierto. Así hasta me evito los problemas de la noche, que como usted sabrá aquí hay mucha fiesta (Gertrudis, comunicación personal, 17 de marzo del 2017).

Gertrudis es un ejemplo de cómo los vínculos pueden conducir a la provisión del cuidado a mujeres indocumentadas. Este caso es una excepción, no se encontraron más casos, pues la mayoría no poseen estas conexiones y por ende no reciben esta información. Gracias a este aprovisionamiento, Gertrudis se gestiona el trabajo y mejora su calidad de vida, se abre camino en medio de las dificultades, ya no tiene que trabajar en las noches, puede usar su tiempo en el día para ocuparse en el mercado laboral

J. Maldonado y el programa Head Start están conectados a través de otro actor privilegiado en la red, S. Jarrín, miembro activo de Caridades Católicas que opera el Head Start en Orleans. Este último recalca que el Apostolado tiene una "red de network" (S. Jarrín, comunicación personal, 24 de abril del 2017) muy eficiente y que está llamada a atraer hispanos hacia el programa, e indica que la iglesia siempre ha estado preocupada por el asunto del cuidado pero que han tenido limitaciones en cuanto a la difusión de sus servicios:

Tenemos un total de cupo para 400, casi 500 niños, después le puedo dar los números ya específicamente. La gran mayoría de los niños que cuidamos son afroamericanos. Los cinco centros están dentro de lo que es la parroquia Orleans. Aquí, en Luisiana, no tenemos Condados, Counties, sino que les decimos Parishes. Entonces, el contrato que tenemos nos permite operar estos centros en Orleans Parish, son cinco. Ahorita no tengo los números, pero es, un pequeño número son hispanos. Creo que el año pasado teníamos quizás un poquito más, pero tuvimos un período de 
transición de un centro a otro. Tuvimos que cerrar uno y abrir otro nuevo y ahí yo creo que perdimos unos cuantos niños pero también hay un sistema que le llaman el One Have, que es el que se está usando, todo el mundo tiene que usar este proceso, para inscribir a los niños en los centros y siempre tenemos muchos retos con ese proceso. Además de los retos normales que tenemos los inmigrantes por el idioma, la cultura, esto y lo otro ¿verdad? Así que el sistema pues tiene que mejorarse y nosotros siempre tenemos que hacer un mejor trabajo de alcance a la comunidad y de dejarle saber a la gente que estamos ahí para servirles (S. Jarrín, comunicación personal, 24 de abril del 2017).

S. Jarrín reconoce que los hispanos que participan del Head Start son quienes han construido algún vínculo con la iglesia y su gestión. Por ejemplo, indica que antes de operar Head Start, con Caridades Católicas: "teníamos un proyecto que se llamaba 'El yoyo'. Ese centro ya no existe, pero los hispanos que estaban ahí, o los niños que estaban ahí cuando lo cerramos, se fueron a los otros centros que teníamos en el área" (S. Jarrín, comunicación personal, 24 de abril del 2017).

Para S. Jarrín, llevar la información a través del "word of mouth, y es que una persona le dice a otra y otra a otra" (S. Jarrín, comunicación personal, 24 de abril del 2017) también es importante en la provisión de este servicio. Indica que de hecho es la forma más efectiva de transmitir la información. Además, concuerda con Ménjivar, Abrego y Schmalzbauer (2016) en que el problema del acceso de los migrantes en los Estados Unidos a beneficios estatales tiene que ver con que especialmente los indocumentados se autolimitan de este recurso por el miedo a la deportación que generan las políticas de seguridad nacional.

S. Jarrín indica, que la institución que él dirige y en general la iglesia católica está creada con la misión de servicio a los demás; y que la eficiencia en la difusión de los bienes que ellos puedan facilitar en la red depende de muchas personas: católicas y no católicas. Es decir, depende de los actores de la red para el cuidado y no solamente de quien opere un programa ofrecido por el gobierno. Estos actores podrían ser: las instituciones que diseñan los programas, los congresistas que aprueban las leyes para aumentar el monto de dinero que se les asignará a estos, etc. Sin embargo, sigue siendo una paradoja que el número de hispanos que son beneficiarios de un programa estatal de cuidado manejado por hispanos de la misma comunidad, sea tan bajo; y solo actoras como Gertrudis, que a modo de estrategia han logrado construir un vínculo fuerte con alguno de estos actores privilegiados pueda aprovisionarse de este bien con relativa facilidad.

Esta experiencia indica que las organizaciones eclesiales son actores de las redes que conectan la comunidad hispana, y que tiene relación con otros actores como el 
Departamento de Educación; ambos se relacionan para proporcionar servicio del cuidado como bien provisto por el Estado. Como ya se indicó, la experiencia de Gertrudis es un ejemplo puntual, en esta investigación no se encontraron más. Sin embargo, debido la variedad de actores que se desenvuelven en la red migratoria con respecto al cuidado, es posible que existan más ejemplos.

\section{Los Contactos Esporádicos}

Los vínculos débiles son entendidos como los "vínculos más casuales y menos estrechos" (Pedone, 2004, p. 337) que se constituyen en "un importante recurso para hacer posible alguna oportunidad" (Granoveter, 1973, p. 1372). Quién posee vínculos débiles "llega a tener poder dentro de las cadenas o redes migratorias" (Pedone, 2004, p. 337) en algún momento, pues amplían el espectro de información en los diferentes espacio que recorren los migrantes. La colección de estos contactos "lejanos" puede significar un aporte importante para el aprovisionamiento del cuidado. El ejemplo de Yajaira indica cómo:

(En el 2016) Me tocó trabajar con doña María M., y doña María M. conoció a la persona que estaba encargada del daycare, como yo estaba dando clases, ella me dijo: mira, lleva a la nena ahí y yo hablo con ella para que ella te ayude. Entonces así hacía. Vamos a decir, yo les limpiaba a ellos los sábados y ahí hacían el cambio, sí. Costaba alrededor de 120 o 130 semanal (Yajaira, comunicación personal, 1 de abril del 2017).

Ella (hija menor de cuatro años) estuvo aquí en la Academy, ahí hice como un trato. Yo limpiaba ahí ese daycare. Yo iba once in a while, así a verla a mi hija. Me gustaba porque ellos hacían muchas actividades (Yajaira, comunicación personal, 1 de abril del 2017).

En sus relatos Yajaira indica que logró aprovisionarse de trabajo y cuidado para su hija a través del establecimiento de un vínculo débil con María M., miembro de la Fundación Golden Change. Ella señala que debido a que María M. contaba con contactos en la institución educativa, pudo interceder para que inscribiera a su hija, pasando por alto algunos protocolos en el proceso de registro.

Yajaira señala que conoció a María M. en unas charlas de apoyo psicológico para mujeres maltratadas que son parte del Programa Federal de Erradicación de la Violencia Intrafamiliar (Family Violence Prevention \& Intervention Program ${ }^{7}$ ) y que son impartidas por

\footnotetext{
${ }^{7}$ Family Violence Prevention \& Intervention Program proviene de un fondo aprobado por la Family Violence Prevention and Services Act (FVPSA)
} 
contratistas como Golden Change. Yajaira asistió como víctima de violencia de género a escuchar estas charlas. En la intervención conoce a María M., a quien posteriormente le pide ayuda para conseguir trabajo.

Más adelante, respondiendo a esta petición, y debido a que María M. tenía urgencia de contratar personal, contacta a Yajaira recordando su buen manejo del idioma inglés, "ella lo habla muy bien" (María M., comunicación personal, 25 de marzo del 2017), y la contrata como maestra de apoyo para el programa de cuidado después de clases "Afterschool Care" para niños del municipio de Metarie, que también opera con su fundación, durante unos meses.

Para que Yajaira pueda desempeñarse en este trabajo, María M. le facilita el contacto con la directora de un centro infantil prestigioso en la ciudad, con quien llega a un acuerdo de intercambio de cuidado por servicios de limpieza. Este es un ejemplo del aprovechamiento de los vínculos débiles con actores privilegiados de la red. Así como los otros ejemplos de actores con autoridad, María M. se ha constituido en un actor privilegiado en la red de la comunidad hispana y facilita bienes como el cuidado dentro ella. María M. busca a Yajaira por "su buen manejo del idioma inglés" e indica que Yajaira llamó su atención porque no es común que las mujeres hispanas en Nueva Orleans, en sus condiciones, logren empezar la secundaria como Yajaira, es decir tener niveles educativos medios y ser fluentes en el idioma inglés.

María M. llega a Nueva Orleans antes del huracán Katrina invitada por su hermana, decide quedarse a probar suerte y logra construir una trayectoria que la destaca en la comunidad hispana. Ella es una migrante con fama en la ciudad que se mueve en diferentes espacios con mucha soltura para crear vínculos ${ }^{8}$. De esta manera, ha logrado ganar contratos estatales como gestora independiente de proyectos con la fundación Golden Change en Nueva Orleans. Esto es un mérito, pues en estos concursos compiten con otras organizaciones como Caridades Católicas, que están apoyadas en instituciones más grandes como la arquidiócesis de la ciudad.

María M. indica que la función principal de Golden Change es el apoyo a las minorías en la ciudad, especialmente a la comunidad hispana. Con respecto al cuidado, opera el programa Afterschool $\mathrm{Care}^{9}$ en algunas instituciones públicas de la metro-área. Ella recibe el material guía de las oficinas del Departamento de Educación de Louisiana en donde se

\footnotetext{
${ }^{8}$ Cuadernos de campo evento Hispanic Chamber of Commerce, 30 de marzo del 2017.

${ }^{9}$ Este programa, funciona en las escuelas públicas pero tiene un costo adicional para los padres que deciden hacer uso de él. El precio tiene una subvención estatal pero se vende en el mercado del cuidado.
} 
le indican los procedimientos y las metas para manejar el programa "todo ya está en papel, solo hay que ponerlo a funcionar" (María M., comunicación personal, 25 de marzo del 2017), y contrata personal por temporadas a medio tiempo, les capacita y les introduce en las escuelas que le son asignadas (María M., comunicación personal, 25 de marzo del 2017).

María M. está y ha estado presente en muchos espacios de la comunidad hispana en la ciudad. Ha sido dirigente barrial, tiene relación con negociantes hispanos dueños de empresas exitosas, tiene contratos con instituciones del gobierno, conoce madres violentadas, hombres maltratadores, niños con deficiencias y tiene relación con la iglesia católica. "A mí me llaman para todo: Doña María M. ¿qué hago? Que ayude a conseguir las estampas, que a apoyar al teenager que le dio un electric shot la policía ¡uf! En fin, estoy en todas" y "todos nos vemos en misa jaja" (María M., comunicación personal, 25 de marzo del 2017). Su trabajo, y cómo ella revela, su personalidad, le han permitido relacionarse con muchos actores de la red y posicionarse como actora privilegiada, acumuladora de capital social y contacto puente para la creación de vínculos.

María M. se encuentra con actoras como Yajaira en la gestión de beneficios del Estado. A pesar de que tiene una oficina en Metarie, su mayor instrumento de contacto es su teléfono celular. Ella indica que el miedo y el desconocimiento de las leyes son las principales razones para que el resto de la población latina no se acerque a estos beneficios y aunque no ha tenido casos concretos con respecto al cuidado, cree que las mujeres evaden el Estado por estas razones pero lo gestionan en otros ámbitos, generalmente en la familia o con cuidadoras no autorizadas.

\section{El acceso a otros programas estatales: soporte para el cuidado}

A pesar de que es poco común que las mujeres indocumentadas se sirvan de los beneficios del Estado para la reproducción de sus vidas y la de sus familias en Estados Unidos, hay algunas que logran informarse, dejan a un lado el miedo y obtienen la asistencia disponible de algunos programas, específicamente para sus hijos nacidos en este país. La información de cuantas mujeres que viven en familias monoparentales con hijos menores de cinco años a su cargo y reciben los subsidios públicos no está desagregada en los censos. Únicamente se conocen los estimados de la ACS (2010-2012) que indican que de 7926 familias hondureñas residentes en Nueva Orleans metro-área en ese período, el 
$15.6 \%$ corresponde a hogares que reciben los beneficios del SNAP ${ }^{10}$ conocido como Food Stamps y únicamente el $4.5 \%$ reciben los beneficios del SSI ${ }^{11}$ (Supplemental Security Income) conocido comúnmente como Disability.

De las entrevistadas, tres: Yajaira, Vanessa y Jasel reciben el beneficio SNAP (Supplemental Nutrition Assistance Program); y una, Yiudmila, recibe el SSI (Supplemental Security Income) para su hija menor discapacitada; ellas son parte de esta minoría. Las razones por la que acceden también se encuentran en sus experiencias migratorias particulares.

A mí me contaron que por mi hija que no tiene su manito y tiene discapacidad yo podía pedir. Pedí aquí, y aquí me la negaron. Yo lo solicite en Tennessee porque aquí estamos muy racistas. Yo lo solicite la primera vez y me dijeron que no podía porque no tenía seguro social y me quede así por mucho tiempo hasta que fui a pasear por Tennessee donde unas familias y una vecina de ellos tenía, y ella no tenía documentos. Ninguno de los dos, ni el esposo, ni la esposa y agarraban su disability. Así que me dijo que vaya. Me dijo: vamos, hablemos con la señora y ella ingresó los datos. Se hizo el registró y me dijo que dentro de un mes usted va a tener una notificación pero que pasee tranquila porque por ahora van a averiguar y eso fue lo que hice. Entonces tuve que pedir traslado de Tennessee para acá y me cancelaron. Yo no estaba de acuerdo con la cancelación, le dije a la señora que yo no podía coger porque no tenía documentos y fue ella la que me indicó que esto no es estatal, que esto es nacional. No tiene que tener privilegio ni para un estado ni para otro, es igual para todos. Me dijo: 'te van a decir que tú tienes que apelar en un juicio entonces tu aceptas, que no te de miedo'. Entonces yo acepté y entonces un juez lo aprobó. Desde ahí lo tengo, desde el 2010 (Yiudmila, comunicación personal, 1 de abril del 2017).

En esta investigación, Yiudmila fue entrevista como proveedora de cuidado (ella es una cuidadora de grupo), y como una de las mujeres cabezas de hogar que a pesar de no tener hijos menores de cinco años, está a cargo de una niña discapacitada que necesita

\footnotetext{
10 EI SNAP (Supplemental Nutrition Assistance Program) es impulsado por el Departamento de Agricultura de los Estados Unidos (USDA). Este es conocido como el Programa de Estampas de Comida que provee beneficios en comida, acceso a una dieta saludable y educación en la preparación de comida y nutrición para las familias de bajos ingresos. Quienes lo reciben deben gastar el beneficio en comprar comida en tiendas autorizadas. La forma de suministro es otorgar una tarjeta electrónica que se usa como una tarjeta del cajero automático para uso exclusivo de alimentos. SNAP es el nombre federal del programa. Sin embargo, los Estados están habilitados para usar diferentes nombres con el mismo propósito. Para reclamarlo se necesita residir en el Estado en el que se lo solicita. Los únicos beneficiarios a este programa pueden ser los ciudadanos estadounidenses o ciertos inmigrantes que sean elegibles. Como requisito se debe cumplir dependiendo del Estado con un nivel de ingreso por debajo de la línea de la pobreza en relación a la cantidad de miembros de la familia. EI SNAP no es para todos, en la oficina de recepción de solicitudes se debe presentar documentación que confirme a su identidad y status legal como son SSN Social Security Number, obligatorio para la realización de este tipo de trámites. (www.benefits.com).

11 Programa de Seguridad de Ingreso Suplementario (SSI, siglas en inglés) que paga beneficios a los adultos y a niños incapacitados que tienen ingresos bajos y recursos limitados (Social Security Administration website).
} 
cuidados especiales durante la mayor parte de día, todos los días. El único servicio público que ella se atreve a requerir del Estado es aquel que se relaciona con la discapacidad de su hija. En su acercamiento a la institución pública que lo otorga, se observa el valioso aporte de los contactos de Yiudmila. Estos le explican desde los procedimientos a seguir hasta las instituciones a donde debe recurrir para obtener el beneficio. Yiudmila consigue el disability para su hija ciudadana estadounidense. En este proceso no solicitan más que una identificación que demuestre que es madre de la niña y una prueba de su discapacidad, por lo que no tuvo complicaciones por portar estatus legal irregularizado. Yiudmila indica que tenía miedo de acercarse a las oficinas del Seguro Social, pues "yo pensaba que si el rato que me pidan los papeles y yo no los tuviera ellos me deportarían". Sin embargo, se sorprende de que exista la posibilidad de la gestión de beneficios del Estado sin pruebas de residencia regularizada de los padres.

Yiudmila indica que obtiene alrededor de $\$ 500$ del disability y lo gasta en las necesidades de su hija, y la mayoría lo usa para pagar a centros de terapias y masajes en donde la reciben unas horas al día. Sin embargo, algunas veces también "toma prestado" este dinero para cubrir otros gastos familiares. Este ingreso le permite cuidar a su hija en su residencia mientras cuida de otros niños y cubrir en resto de gastos.

De la misma manera, Yajaira indica que sus contactos, como el de María M., le compartieron información de los posibles inconvenientes de la aplicación a beneficios estatales en trámites futuros. Por ejemplo, Yajaira indica que ella únicamente aplicó a SNAP, debido a que por contar con TPS ${ }^{12}$ (desde hace un par de años) tenía la esperanza de aplicar al permiso de residencia permanente en algún momento ${ }^{13}$, y le han comentado que si aplica a otros beneficios, está posibilidad se anularía: "si te dan ya puedes entrar como carga $^{14}$ y eso te perjudica en la pedida de los papeles" (Yajaira, comunicación personal, 1 de abril del 2017).

12 Temporary Protected Status/ Estatus de Protección Temporal) fue un fuerte golpe para algunos grupos hispanos. EI TPS era una medida de protección temporal que el secretario del Departamento de Seguridad Nacional activaba para los originarios de un país extranjero que no garantizaba a sus ciudadanos regresar a su país de manera segura (USCIS 2018). La decisión de cancelación del TPS para hondureños ${ }^{12}$ se anunció en mayo del 2018, luego de anunciar la apertura del pre registro de la última renovación de ese beneficio que terminará el 5 de enero del 2020. Luego de esa fecha, los hondureños que estaban amparados bajo el TPS no podrán residir en los Estados Unidos (USCIS 2018).

${ }^{13}$ En el momento de la entrevista todavía no se había cancelado el TPS para los hondureños. Meses después el presidente Trump envía la cancelación que indica que migrantes como Yajaira tienen el plazo máximo hasta inicios del 2021 para salir de Estados Unidos. Con esta cancelación su esperanza de ser residente legal queda anulada.

${ }^{14}$ Carga pública: término que usan las entidades del Estado para denominar a las personas que podrían depender del gobierno como su fuente principal de manutención (USCIS 2017). 
Como indica esta entrevistada esta información está desplegada en las redes, los migrantes la conocen y la comparten con los integrantes más cercanos "de boca en boca". Las personas que no cuentan con vínculos que les provean esta información generalmente caen en esta categorización y tienen inconvenientes futuros en los procesos de regularización.

En la web no te indican que tienes consecuencias si agarras más bonos. Te dicen vea usted que hace, pero la gente te cuenta sus historias de lo que ha sido con ellos. Entonces yo conozco a esta mujer que no ha podido conseguir los papeles, la green card pues por haber agarrado el cash (Yajaira, comunicación personal, 1 de abril del 2017).

Pese a que Yajaira es una mujer educada, que habla bien inglés y que usa en forma eficiente las herramientas tecnológicas como el internet, también hace uso de sus contactos para conseguir beneficios. Ella provee la siguiente información sobre el proceso:

Los requisitos (para obtener SNAP) son tener un ingreso bajo, tener niños que son nacidos acá en los Estados Unidos, a veces algunos mentimos. Lo necesitamos pero a veces los gobiernos te hacen mentir por el hecho de que ellos no entienden. Por el hecho de que estas trabajando ya alcanza, no es así. No miran el aspecto de los libros, la luz, el carro, la ropa. Osea la visión de ellos es no más lo que ganes. Lo otro, ya a ellos no les interesa, ya te acomodas o te acomodas (Yajaira, comunicación personal, 1 de abril del 2017).

Cada seis meses tienen que llenar unos papeles indicando que si tus ingresos subieron, bajaron, si vives en la misma casa, si es tuya. Osea son muchas cosas. Después de las elecciones del presidente Trump van inspectores. Fueron a una casa que yo había puesto antes en donde yo vivía pero no quería cambiar de dirección porque a veces cuando cambias de dirección en la mitad del proceso, te cortan lo que es la manutención, lo que te dan el dinero. Entonces, yo no lo quería hacer y me contaron que fueron a verificar. Si no eres legal, mientras el niño sea legal igual te dan. Ahora, si el niño no es ciudadano americano no te dan (Yajaira, comunicación personal, 1 de abril del 2017).

Te dan como una tarjeta de crédito $^{15}$, es dependiendo, es dependiendo cada niño. A mí por mis hijos me están dando aproximadamente $\$ 550$. La gente lo mira mucho, pero no es mucho porque lo que es en la carne, el arroz, los huevos, la leche, golosinas para ellos, su desayuno. Ahí solo se van casi como \$200 (Yajaira, comunicación personal, 1 de abril del 2017).

Yajaira destaca que ella no cumple con el requisito de percibir ingresos menores a la línea de la pobreza, pero que recibe este beneficio del Estado debido a la alteración de su información en la aplicación. Declara que esta también es una estrategia común entre los aplicantes a los programas. Yajaira usa el ingreso que viene de estos beneficios para cubrir los gastos de alimentación y "las golosinas". Con estos gastos cubiertos, logra ahorrar

${ }^{15}$ La tarjeta de crédito a la que se refiere Yajaira es la Electronics Benefits Transfer Card (EBT). 
algo de sus ingresos del trabajo para cubrir cuestiones como "lo que le doy a la vecina, si algún día salgo y me los cuida" (Yajaira, comunicación personal, 1 de abril del 2017), por ejemplo; o las actividades recreativas que ella dice realizar sola o con su familia.

De la misma manera, Vanessa recibe beneficios estatales por estar conectada a una red que le provee información. El espacio de trabajo se convierte para ella en un lugar de encuentro para la circulación de información útil para el aprovisionamiento de cuidado y otros beneficios del Estado. Una fuerte relación de amistad con una compañera del trabajo que inicio por "ser latinas" (compartir el idioma), especialmente le ha abierto estas nuevas vías de aprovisionamiento.

En el diario compartir nos conversamos de todo. Hasta que yo expliqué que no tenía como andar con ambos. Estaban ambos en una casa en donde los cuidaban pero no me gustaba porque lo tenían con otros niños y hasta hubo un accidente por el motivo que los grandes son traviesos y ya no me lo querían recibir. Entonces me dijo que 'oye que en la escuela pública empiezan desde los 4, entonces me acerqué a una escuelita, pero no pude pues por donde vivía. Ella mismo me dijo que venga que la vecina está arrendando, y los cogí a mis niños y nos cambiamos para allá (Vanessa, comunicación personal, 18 de abril del 2017).

Vanessa indica que se acerca a la escuela pública que le recomienda su compañera de trabajo Lucy para recibir cuidado de su hijo de 4 años. Allí le informan que su aplicación debe ser en línea. Ella no entiende este proceso, por lo que requiere asistencia y le envían a un Centro de Información organizado por el Departamento de Educación. En ese lugar le ayudan a aplicar al programa, pero su aplicación no despliega ningún servicio dispuesto para el sector de su domicilio. Vanessa frustrada recurre a su amiga nuevamente, y ella le recomienda mudarse al barrio en donde ésta asegura que existe el servicio.

Vanessa indica que a través de esta mudanza y el impulso de Lucy para aplicar logró inscribir a tiempo a su hijo. Ella no conoce el nombre del programa pero podría tratarse del Title I o LA4, que son programas que ofrecen cobertura de cuidado y educación temprana a niños de 4 años en algunas escuelas públicas. Estos programas están dispuestos en sectores de la ciudad que albergan población vulnerable. Por tanto quienes viven lejos pueden aplicar, pero el sistema da preferencia a quienes están cerca. Generalmente los cupos se llenan con aplicantes del barrio. Esta también es una muestra de cómo las mujeres van tejiendo redes en los espacios por donde transitan y que los vínculos que van construyendo a su paso les ayudan a acercarse al aprovisionamiento de algún bien o servicio (acumulan capital social). Lo mismo pasa con la provisión de la tarjeta Food Stamp 
(SNAP). Lucy le anima a aplicar, Vanessa aplica y con el ingreso de este beneficio "se ayuda" y cubre los gastos de alimentación e igual que a Yajaira y Yiudmila esto le permite usar sus ingresos del trabajo para otros gastos.

Por su parte, Jasel explica que por vivir alrededor de 10 años en Estados Unidos y contar con el consejo de familiares que llegaron antes (son autoridad) que conocían los beneficios, aplicó al SNAP en Miami poco después del nacimiento de su primer hijo. Ella declara que:

\begin{abstract}
Es una gran ayuda para las madres solas. Aquí la comida es muy cara. Yo tengo tres hijos y por más que estiro el gasto no alcanza. Con la tarjeta se hace más fácil, y lo bueno es que la cogen en todos lados, tanto en Walmart como en otros supermercados. Lo malo es que solo paga la comida. A veces se encuentra lugares más convenientes pero como no es efectivo no se puede pagar (Jasel, comunicación personal, 8 de abril del 2017).
\end{abstract}

Para Jasel el beneficio que le otorga la tarjeta de Food Stamp es muy importante, pues aporta considerablemente a la cobertura de los gastos familiares. Ella es una madre soltera con tres hijos a su cargo y no alcanza a sostener a su familia con el ingreso del trabajo con el que cuenta. Jasel es ayudante de cocina en un emprendimiento latino del que percibe una remuneración semanal muy baja, a cambio de empezar la jornada muy temprano en la mañana, este no es un salario constante debido a que indica que muchas veces su jefa le ha descontado una cantidad significativa por no presentarse a trabajar por algún percance familiar o por llegar tarde.

Hay días que no puedo con todo, se enferma el uno y les contagia a los demás. Aquí hay esto de que no pueden ir a la escuela si tienen fiebre. Eso me ha acabado. Si me entiende. Yo no tengo con quien encargarlos, en el barrio es peligroso y si los dejo me los pueden quitar. He oído de casos de mamás que los dejan dormidos y se los llevan, y sin papeles, no los vuelven a ver. A veces los he llevado conmigo al trabajo, pero a la señora no le gusta. Ella como que piensa que los niños le ahuyentan, le ensucian, y si son traviesos (Jasel, comunicación personal, 8 de abril del 2017).

Jasel indica que en algunas ocasiones estuvo tan urgida de dinero que hizo negocios con su tarjeta de estampas de comida: "Yo le ofrecí a ella que si, que si le interesaría que intercambiemos, que yo le compro la comida con la tarjeta y ella me devuelve el cash" (Jasel, comunicación personal, 8 de abril del 2017). Jasel señala cuando ha conseguido realizar estas transacciones, ha usado el dinero para pagarle a la señora que cuida a su 
hija $^{16}$. Destaca que luego de pagar la renta y algunos servicios ya no le queda dinero en efectivo para cubrir el resto de los gastos y recurre a esta estrategia para suplir sus necesidades. Jasel y sus tres hijos viven en un departamento independiente de un solo ambiente (estudio) que es parte de una casa divida en varios departamentos.

Esta mujer es la segunda más educada de las siete entrevistadas. Sin embargo, es la que más complicaciones diarias tiene para reproducir su vida en los Estados Unidos. A pesar de que sus hijos mayores atienden a la escuela pública y que ha conseguido aprovisionarse del cuidado de su hija menor por las mañanas, ella no tiene tiempo para trabajar por las tardes y no cuenta con una red de apoyo constante por lo que no tiene posibilidad de mejorar sus ingresos.

De la tarjeta no se puede vivir. Yo que ya tengo tres hijos tengo que dedicarme a otros trabajos. No se cómo hacen otras que ya se cruzan de brazos. Yo me dedico a trabajar el tiempo que tengo. El único servicio que he agarrado es el Food Stamp, creo que siempre he sido una mujer luchadora, hay veces que tenemos cosas y no sabemos apreciar pero cuando te toca una vida dura ahí te toca luchar trabajar, y buscar una responsabilidad (Jasel, comunicación personal, 8 de abril del 2017).

\section{CONCLUSIONES}

En la comunidad hispana de New Orleans los hondureños van tejiendo redes que se conectan con respecto al idioma, la religiosidad o la pasión por el deporte, por ejemplo. Estas redes se van ampliando con el tiempo. Cada hondureño/a que llega a New Orleans tiene la oportunidad de aprovechar algún bien que se encuentre dispuesto en ellas. Y, la posibilidad de acceder a las bondades de estas redes, de acuerdo con esta tesis, depende de la aptitud para moverse en ellas, ampliar contactos, crear vínculos, o acumular capital social; capitalizarse.

Las mujeres entrevistadas ingresan en New Orleans con información y contactos limitados que les permiten aprovisionarse de bienes como el trabajo y la vivienda. Sin embargo, a través de la estrategia principal de ir creando vínculos con actores influyentes en la red o que cuenten con privilegios, experiencia o información; logran insertarse y moverse en medio de estas redes para aprovisionarse del cuidado, que se constituye en un bien indispensable para la reproducción de sus vidas.

${ }^{16}$ Cabe resaltar que la hija de Jasel recibe el cuidado de una "cuidadora de grupo". 
La iglesia católica tiene mucha fuerza en la comunidad hispana de la ciudad. Es por ello que la estrategia de las mujeres consiste en la creación de un vínculos con sus miembros o con quienes tienen vínculos a estos miembros para poder conducirse hacia las instituciones públicas y privadas que se alinean con respecto al cuidado, así como también las conducen al mercado con transacciones diferentes al pago en efectivo a cambio del servicio, y a otras personas e instituciones que también se encuentran desplegadas en la red del cuidado para las mujeres migrantes como las oficinas del Seguro Social que facilita beneficios públicos en forma de bonos a los que algunas acceden, y por medio de los cuales generan ingresos para el cuidado.

\section{REFERÊNCIAS}

Child Care Aware of America. Child Care in America: 2014 State Fact Sheets. Virginia: Child Care in America, 2014.

Euraque, Samantha. Honduran memories": identity, race, place and memory in New Orleans, Louisiana. New Orleans: Luisiana State University, Master of Arts (MA) Geography and Anthropology, 2004.

Granovetter, Mark. The Strength of Weak Ties. Chicago, The American Journal of Sociology, 1973. p. 1360-1380.

Murillo, Indira. Características Sociodemográficas de las Mujeres Migrantes Hondureñas en Estados Unidos de América, Período 2001- 2013. Tegucigalpa: Revista Población y Desarrollo: Argonautas y Caminantes, 2016. p. 53-63.

Menjívar, Cecilia, Leisy Abrego, y Leah Schmalzbauer. Immigrant Families. Inmigration and society. Cambridge: Polity Press, 2016.

Narotzky, Susana. El lado oculto del consumo. Cuadernos de Antropología Social, $\mathrm{n}^{\circ}$ 26, p. 21-39. 2007.

Pedone, Claudia. Cadenas y Redes migratorias: propuesta metodológica para el análisis diacrónico- temporal de los procesos migratorios. Madrid: Empiria, octubre 2010.

Pedone, Claudia. Tú siempre jalas a los tuyos. Cadenas y redes migratorias de las familias hacia España. Tesis doctoral. Barcelona: Universitat Autònoma de Barcelona, 2004.

Pérez Orozco, Amaia. Miradas globales a los cuidados del desarrollo: ¿Por un derecho al cuidado? . Santo Domingo: INSTRAW, 2009.

Portes, Alejandro. «Immigration Theory for a New Century: Some Problems and Opportunities.» En The Handbook of International Migration: The American Experience, de 
P. Kasinitz y J. DeWind. Editado por C. Hirschman, 1-33. New York: Russell Sage Foundation, 1999.

Pozas, María de Los Ángeles. Sociología económica y migración internacional: convergencias y divergencias. En El país trasnacional. Migración mexicana y cambio social a través de la frontera, de Coordinador por: Marina Ariza y Alejandro Portes, 619650. México, DF: UNAM-Instituto de Investigaciones Sociales, 2007.

Vega, Cristina. Culturas del cuidado en transición. Espacios, sujetos e imaginarios en una sociedad de migración. Barcelona: UOC, 2009. 